\title{
LEAST-PERIMETER PARTITIONS OF THE DISK INTO THREE REGIONS OF GIVEN AREAS
}

\author{
ANTONIO CAÑETE AND MANUEL RITORÉ
}

\begin{abstract}
We prove that the unique least-perimeter way of partitioning the unit 2-dimensional disk into three regions of prescribed areas is by means of the standard graph described in Figure 1
\end{abstract}

\section{INTRODUCTION}

Partitioning problems in the Calculus of Variations have multiple applications in physical sciences. They can model multitude of natural phenomena such as the shape of a cellular tissue, the interface of separation between fluids, and many others, as described in the treatise by D'Arcy Thompson [Th].

In this work we consider the isoperimetric problem of partitioning a planar disk into three regions of given areas with the least possible perimeter, and we prove that the standard configuration in Figure 1, consisting of three circular arcs or segments meeting orthogonally the boundary of the disk, and meeting in threes at 120 degrees in an interior vertex, is the only solution to this problem.

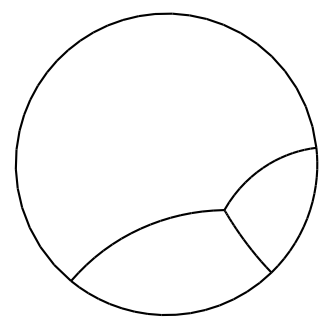

FiguRE 1. The least-perimeter partition of the disk into three given areas

In addition to the above conditions, the solution must satisfy a certain balancing condition on the geodesic curvatures of the circles. This condition will be stated precisely in the next section.

Existence and regularity of solutions for this problem are guaranteed by the results of F. Morgan [M2, who showed that the minimizer, in the interior of the disk, is composed of smooth curves of constant geodesic curvature meeting in threes at 120 degree angles.

Date: June 25, 2003.

2000 Mathematics Subject Classification. 49Q10, 51M25, 52A38, 52A40.

Key words and phrases. Isoperimetric partition, stability, stable.

Both authors have been supported by MCyT-Feder research project BFM2001-3489. 
Boundary regularity also follows from $\mathbf{M 2}$ although it is not explicitly stated in his work. Existence and regularity in higher dimension were studied by F. Almgren $\mathbf{A l m}$.

The least-perimeter way of partitioning a disk $D$ into two regions of given areas is by means of an arc of circle or segment that meets orthogonally $\partial D$. From the existence and regularity results in next section it follows that there is a solution, which is a smooth, possibly nonconnected, embedded curve with constant geodesic curvature that meets $\partial D$ orthogonally. Such a curve must be connected, since otherwise we could rotate one component with respect to the center of the disk until it touches a second one, thus producing a non-allowed singularity. On the other hand, as the curve has constant geodesic curvature, it must be part of a circle or of a line.

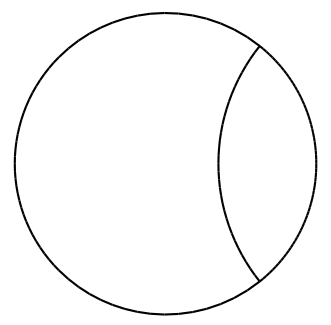

FiguRE 2. The least-perimeter partition of the disk into two given areas

The isoperimetric problem consisting of enclosing $n$ given areas in the disk or in the plane with the least possible perimeter has a strong complexity which is derived not from the geometry of the individual components of the solution (they can be described in terms of circles or lines) but from their large number.

The planar double bubble conjecture was proved by J. Foisy et al. [FABH], who showed in 1993 that the standard planar double bubble uniquely minimizes perimeter in $\mathbb{R}^{2}$. Assuming that the studied regions are connected, C. Cox et al. [CHH] proved in 1994 that the standard planar triple bubble uniquely minimizes perimeter in the plane for any three given areas. R. P. Devereaux [D] studied in 1998 the planar triple bubble conjecture under the hypothesis that all the regions have the same pressure. W. Wichiramala finally proved the planar triple bubble conjecture in 2002 in his Ph. D. Thesis [W]. J. Masters $\mathbf{M}$. proved in 1996 the double bubble conjecture in $\mathbb{S}^{2}$. Interesting preliminary work was carried out by Bleicher [B1, B2, B3. Concerning boundary problems, G. Hruska et al. HLPS have obtained some results for planar bubbles in corners. Also results on tori and cones have been obtained in CHLL, BL.

In higher dimensions, J. Hass and R. Schlafly [HS proved the double bubble conjecture in $\mathbb{R}^{3}$ for equal volumes. The general conjecture was settled by M. Hutchings et al. HMRR. For higher dimensional Euclidean spaces Reichardt et al. RHLS have obtained a proof of the double bubble conjecture in $\mathbb{R}^{4}$ and partial results in higher dimensional Euclidean spaces. In the three-dimensional torus, M. Carrion et al. CCWB] have provided numerical evidence for a double bubble conjecture with ten types of solutions. In the three-dimensional sphere and the three-dimensional hyperbolic space, A. Cotton and D. Freeman $[\mathbf{C F}$ have also obtained partial results on the conjecture that the standard double bubble in these spaces uniquely minimizes perimeter. 
Planar bubbles are also of great interest to physicists. Interesting articles focusing on physical aspects of the problem are GJJF and CGFM.

The most interesting mathematical open question for these problems is to show that the minimizing configurations must have connected regions, either in $\mathbb{R}^{2}$ or in the disk. In addition, in the planar problem, one should also be able to prove that the exterior region is connected, i.e., that there are no empty chambers.

We have organized this paper in several sections. In Section [1 we give precise definitions, compute the first and second variations of length for graphs, recall existence and regularity results for the problem of minimizing perimeter while partitioning the disk into given areas, and state some properties which minimizing graphs must satisfy. In Section 2 we obtain a bound on the number of components of the largest pressure region determined by a graph which minimizes perimeter up to second order. We conclude that a minimizing configuration must have one of ten possible types, described in Figure 4. In Section 3 we prove the necessary results to discard the possibilities obtained in Section 2, which allow us to prove our Main Theorem in Section 4. In a final section, we indicate further lines of research and give several conjectures.

All the pictures in this paper have been made by using Surface Evolver, a software developed by Ken Brakke (http://www.susqu.edu/facstaff/b/brakke/).

\section{Preliminaries}

1.1. Notation. Let $D \subset \mathbb{R}^{2}$ be the closed unit disk in centered at the origin. An admissible graph $C \subset D$ consists of vertices and curves so that at every interior vertex (that is, a vertex in the interior of $D$ ) three curves of $C$ meet and at every boundary vertex (a vertex in $\partial D$ ) just one curve of $C$ meets $\partial D$. We shall also assume that $C$ induces a decomposition of the open unit disk into $n$ regions $R_{i}, 1 \leqslant i \leqslant n$, possibly nonconnected. An m-component is a connected component of a region with $m$ edges.

If $R_{i}$ and $R_{j}$ are adjacent regions, we will denote by $C_{i j} \subset C$ the (not necessarily connected) curve separating them. Let $I(i)=\left\{j \neq i ; R_{j}\right.$ touches $\left.R_{i}\right\}$. With this notation

$$
\partial R_{i} \cap \operatorname{int}(D)=\bigcup_{j \in I(i)} C_{i j}
$$

We shall denote by $N_{i j}$ the normal vector to the curve $C_{i j}$ pointing into the region $R_{i}$, and by $h_{i j}$ the geodesic curvature of the curve $C_{i j}$ with respect to the normal $N_{i j}$.

A standard graph consists in three circular arcs or lines segments meeting at an interior vertex at 120 degree angles, reaching orthogonally $\partial D$, and so that the sum of the geodesic curvatures is zero.

Given $n$ positive numbers $a_{1}, \ldots, a_{n}$ such that $\sum_{i=1}^{n} a_{i}=\pi$, the isoperimetric profile is the function $I\left(a_{1}, \ldots, a_{n}\right)$ defined as the infimum of the lengths of all admissible graphs separating regions in the disk of areas $a_{1}, \ldots, a_{n}$.

We will say that an admissible graph $C$ is minimizing for prescribed areas $a_{1}, \ldots, a_{n}$ if $I\left(a_{1}, \ldots, a_{n}\right)$ is attained by $C$. 
1.2. Variational formulae. Given an admissible graph $C \subset D$, we will consider smooth one-parameter variations $\varphi_{t}: C \rightarrow D$ for $t$ small, which satisfy $\varphi_{t}(\partial D) \subset \partial D$. We will denote by $X=d \varphi_{t} /\left.d t\right|_{t=0}$ the associated infinitesimal vector field, which is smooth on every curve $C_{i j}$. Note that $X(p)$ is tangent to $\partial D$ for each $p$ in $\partial D$. Let $u_{i j}=X \cdot N_{i j}$ be the normal component of $X$ on $C_{i j}$.

Given such a variation, it is easy to check that the derivative of the area $A_{i}$ of $R_{i}$ at $t=0$ is given by

$$
\left.\frac{d A_{i}}{d t}\right|_{t=0}=-\sum_{j \in I(i)} \int_{C_{i j}} u_{i j} .
$$

For the derivative of length for such a variation we have

Proposition 1.1 (First variation of length HMRR, Lemma 3.1]). Consider an admissible graph $C \subset D$, and a smooth variation $\varphi_{t}: C \rightarrow D$ with associated vector field $X$. Then the first derivative of the length of $\varphi_{t}(C)$ at $t=0$ is given by

$$
\left.\frac{d L}{d t}\right|_{t=0}=-\frac{1}{2} \sum_{\substack{i \in\{1, \ldots, n\} \\ j \in I(i)}}\left\{\int_{C_{i j}} h_{i j} u_{i j}+\sum_{p \in \partial C_{i j}} X(p) \cdot \nu_{i j}(p)\right\},
$$

where $\nu_{i j}(p)$ is the inner conormal to $C_{i j}$ in $p$.

We will say that an admissible graph is stationary if (1.2) vanishes for any area-preserving variation. From Proposition 1.1 it is easy to prove the following

Proposition 1.2. Let $C \subset D$ be a stationary graph. Then the following conditions are satisfied

(i) The geodesic curvature $h_{i j}$ is constant on $C_{i j}$.

(ii) The edges of $C$ meet in threes at 120-degree angles in interior vertices.

(iii) The balancing condition: three edges $C_{i j}, C_{j k}, C_{k i}$ meeting in an interior vertex satisfy

$$
h_{i j}+h_{j k}+h_{k i}=0 \text {. }
$$

(iv) The edges of $C$ meet $\partial D$ orthogonally at boundary vertices.

Condition (ii) implies that, in some interior vertex where the three curves $C_{i j}, C_{j k}, C_{k i}$ meet, the normals add up to zero, i. e., $N_{i j}+N_{j k}+N_{k i}=0$. This implies that the normal components of the vector field $X$ must satisfy

$$
u_{i j}+u_{j k}+u_{k i}=0 .
$$

Given a stationary graph $C$, and a function $u: \bigcup_{i, j} C_{i j} \rightarrow \mathbb{R}$, with $u_{i j}=\left.u\right|_{C_{i j}}$, satisfying condition (1.4) on every interior vertex, it is always possible to find a vector field $X$ on $C$, so that $u_{i j}=X \cdot N_{i j}$ and $X$ is tangent to $\partial D$ in each boundary vertex. Associated to $X$ one can also find a one-parameter variation $\varphi_{t}: C \rightarrow D$, for $t$ small enough, so that $\varphi_{t}(p)=\exp _{p}(t X(p))$ for any $p$ out of an arbitrarily small neighbourhood of $\partial D$. The argument is as follows: fix an arbitrary neighbourhood $U$ of $\partial D$ that does not contain interior vertices of $C$. Modify $X$ so that it is normal to $C$ in $U$. Let $\nu$ be the inner normal to $\partial D$. Extend it to $U$ so that it is tangent to the edges of $C$. Also extend $X$ to a vector field on $U \cap D$ by means of the exponential mapping. Let $\lambda$ be a smooth function equal to 1 near $\partial D$ with 
support in $U$. Consider the vector field $Y=X-(X \cdot(\lambda \nu)) \lambda \nu$ and the local one-parameter group $\psi_{t}$ generated by $Y$. Since $\nu$ is tangent to $C$ and $X$ is normal to $C$ in $U$, we have that $Y=X$ on $C$. Moreover, for $p \in \partial D$, the vector $Y(p)$ is tangent to $\partial D$. Hence the deformation $\psi_{t}(C \cap U)$ has initial velocity vector field $X$ and keeps $C$ inside the disk. The variation $\psi_{t}(C)$ has the further property that coincides with $\exp _{p}(t X(p))$ in $U$ out of the support of $\lambda$. Now we simply define $\varphi_{t}(p)$ equal to $\exp _{p}(t X(p))$ out of the support of $\lambda$, and equal to $\psi_{t}(p)$ in $U$.

The balancing condition (1.3) allows us to define a pressure $p_{i}$ on every region $R_{i}$, starting from a given region, so that

$$
h_{i j}=p_{i}-p_{j} .
$$

These pressures are determined up to an additive constant. The first variation formula of length can be rewritten in terms of pressures in the following way: if $C$ is a stationary graph, then the first variation of length for an arbitrary variation is given by:

$$
\frac{d L}{d t}=\sum_{i=1}^{n} p_{i} \frac{d A_{i}}{d t}
$$

Observe that the indetermination of the pressures up to some additive constant does not affect the above formula since $\sum_{i=1}^{n} d A_{i} / d t=0$ for any variation of the regions $R_{i}$, as $\sum_{i=1}^{n} A_{i}(t)=$ area $(D)$ along the variation.

Let us prove now the second variation formula of length

Proposition 1.3 (Second variation of length). Let $C$ be a stationary graph and let $\left\{\varphi_{t}\right\}$ be a variation with associated vector field $X$ preserving areas up to second order. Then the second derivative of length at $t=0$ is given by

$$
\begin{aligned}
-\frac{1}{2} \sum_{\substack{i=1, \ldots, n \\
j \in I(i)}}\left\{\int_{C_{i j}}\left(u_{i j}^{\prime \prime}+h_{i j}^{2} u_{i j}\right) u_{i j}+\sum_{\substack{p \in \partial C_{i j} \\
p \in \operatorname{int}(D)}}\left(-q_{i j} u_{i j}^{2}+u_{i j} \frac{\partial u_{i j}}{\partial \nu_{i j}}\right)(p)\right. \\
\left.+\sum_{\substack{p \in \partial C_{i j} \\
p \in \partial D}}\left(u_{i j}^{2}+u_{i j} \frac{\partial u_{i j}}{\partial \nu_{i j}}\right)(p)\right\},
\end{aligned}
$$

where $q_{i j}(p)=\left(h_{k i}+h_{k j}\right)(p) / \sqrt{3}$, and $R_{k}$ is the third region touching the vertex $p$.

Proof. Differentiating the integral terms in equation (1.2), we get

$$
\left.\frac{d}{d t}\right|_{t=0}\left(\int_{C_{i j}} h_{i j} u_{i j}\right)=\int_{C_{i j}}\left(u_{i j}^{\prime \prime}+h_{i j}^{2} u_{i j}\right) u_{i j}+\left.h_{i j} \frac{d}{d t}\right|_{t=0}\left(\int_{C_{i j}} u_{i j}\right),
$$

but since the variation preserves areas up to second order, it follows that

$$
\left.\sum_{\substack{i \in\{1, \ldots, n\} \\ j \in I(i)}} h_{i j} \frac{d}{d t}\right|_{t=0}\left(\int_{C_{i j}} u_{i j}\right)=\left.2 \sum_{i=1}^{n} p_{i} \frac{d^{2} A_{i}}{d t^{2}}\right|_{t=0}=0 .
$$

Differentiating now the second term in equation (1.2), we get

$$
\left.\frac{d}{d t}\right|_{t=0}\left(X \cdot \nu_{i j}\right)=\left(D_{X} X \cdot \nu_{i j}\right)+u_{i j} h_{i j}\left(X \cdot \nu_{i j}\right)+u_{i j} \frac{\partial u_{i j}}{\partial \nu_{i j}} .
$$


For $p \in \operatorname{int}(D)$, the first term vanishes since $\nu_{i j}+\nu_{j k}+\nu_{k i}=0$, and after some calculations as in [HMRR], the second one can be seen as $-q_{i j} u_{i j}^{2}$, where $q_{i j}=\left(h_{k i}+h_{k j}\right) / \sqrt{3}$. For $p \in \partial D$, since the configuration is stationary, the edges meet $\partial D$ orthogonally, so that $D_{X} X(p) \cdot \nu_{i j}(p)$ equals $u_{i j}^{2}$ times the geodesic curvature of $\partial D$, and $\left(X \cdot \nu_{i j}\right)(p)=0$.

The condition that the variation must preserve area up to second order is not really needed as we can show in the next Lemma

Lemma 1.4. Let $C \subset D$ be a stationary graph. Given smooth functions $u_{i j}: C_{i j} \rightarrow \mathbb{R}$ such that (1.1) and (1.4) are satisfied (a variation that preserves area up to first order is given), there is a variation $\left\{\varphi_{t}\right\}$ of $C$ which leaves constant the area of the regions enclosed by $\varphi_{t}(C)$ and such that the normal components of the initial velocity vector field $X$ are the functions $u_{i j}$.

Proof. Let $X$ be a vector field on $C$, smooth over each curve $C_{i j}$, such that $X \cdot N_{i j}=u_{i j}$. Let $\psi_{t}: C \rightarrow D$ be a one-parameter variation of $C$ associated to $X$ such that $\psi_{t}(p)=\exp _{p}(t X(p))$ out of a small neighbourhood $U$ of $\partial D$ which does not contain interior vertices of $C$.

We label the regions $R_{i}$ so that $R_{i}$ touches $R_{i+1}$ for $i=1, \ldots, n-1$. Choose positive functions $v_{i}$ with support in the interior of $C_{i(i+1)}$ and out of $U$. The variation induced by the vector field $v_{i} N_{i(i+1)}$ decreases the area of $R_{i}$, increases the area of $R_{i+1}$ and leaves constant the area of the remaining regions.

Consider the variation equal to

$$
\left(t, s_{1}, \ldots, s_{n-1}\right) \longmapsto \exp _{p}\left(t X(p)+\sum_{i=1}^{n-1} s_{i} v_{i} N_{i(i+1)}(p)\right), \quad \text { in } C \cap(D-U),
$$

and equal to $\psi_{t}(p)$ for $p \in C \cap U$. Consider the function $\left(A_{1}, \ldots, A_{n-1}\right)$ of $\left(t, s_{1}, \ldots, s_{n-1}\right)$, given by the areas of the deformation of the regions $R_{1}, \ldots, R_{n-1}$. The Jacobian

$$
\frac{\partial\left(A_{1}, \ldots, A_{n-1}\right)}{\partial\left(s_{1}, \ldots, s_{n-1}\right)}
$$

is lower triangular, with non-vanishing entries in the principal diagonal, so that the matrix is regular. The Implicit Function Theorem allows us to find smooth functions $s_{1}(t), \ldots, s_{n-1}(t)$ such that $A_{i}\left(t, s_{1}(t), \ldots, s_{n-1}(t)\right)$ is constant for all $i$.

The initial velocity vector field of such a variation is equal to $X$ on $C \cap U$, and to $X+$ $\sum_{i=1}^{n-1} s_{i}^{\prime}(0) v_{i} N_{i(i+1)}$ on $C \cap(D-U)$. As $s_{i}^{\prime}(0)=0$ since $\psi_{t}$ preserves areas up to first order, we conclude that $X$ is the initial velocity vector field.

Remark 1.5. A variation of a stationary graph $C$ by stationary graphs preserves the angles between edges at interior vertices and the orthogonality condition at boundary vertices. Given a variation preserving the area of all the regions up to first order, we can modify it by Lemma 1.4 so that the areas enclosed are constant along the deformation. From the second variation formula we get that the second derivative of length is given by

$$
\frac{d^{2} L}{d t^{2}}=\sum_{\alpha} \frac{d p_{\alpha}}{d t} \frac{d A_{\alpha}}{d t}
$$

where $\alpha$ labels the components of the stationary graph (regions can be disconnected), and $d p_{\alpha} / d t$ is the derivative of the pressure of the component $\alpha$ with respect to the considered variation. Take into account that the quantity $u_{i j}^{\prime \prime}+h_{i j}^{2} u_{i j}$, the derivative of the geodesic 
curvature $h_{i j}$, only depends on $u_{i j}$, the normal component of the variational vector field $X$, and that the modification needed in Lemma 1.4 to preserve areas only modifies the acceleration of the variation. The angle-preserving condition depends only on the initial velocity vector field.

In general, if the areas are not preserved up to second order, the second derivative of length, for a deformation of a stationary graph by stationary graphs, is given by

$$
\frac{d^{2} L}{d t^{2}}=\sum_{\alpha} \frac{d p_{\alpha}}{d t} \frac{d A_{\alpha}}{d t}+p_{\alpha} \frac{d^{2} A_{\alpha}}{d t^{2}}
$$

which can also be obtained by differentiating equation (1.6).

Remark 1.6. For a variation such that the angles between the edges are preserved, we have $D_{X}\left(\nu_{i j}+\nu_{j k}+\nu_{k i}\right)=0\left(\right.$ since $\nu_{i j}+\nu_{j k}+\nu_{k i}=0$ for all $\left.t\right)$, so the boundary term in the second variation formula vanishes.

1.3. Admissible functions and the index form. Let $C$ be a stationary graph. We say a function $u: \bigcup_{i, j} C_{i j} \rightarrow \mathbb{R}$ is admissible if the restrictions $u_{i j}=\left.u\right|_{C_{i j}}$ lie in the Sobolev space $W^{1,2}\left(C_{i j}\right)$, and verify that at any interior vertex $p, u_{i j}(p)+u_{j k}(p)+u_{k i}(p)=0$. These functions correspond to variations of $C$ which have as normal components of the associated vector field $X$ the functions $u_{i j}$. These variations will preserve areas if, for each $i$,

$$
\sum_{j \in I(i)} \int_{C_{i j}} u_{i j}=0
$$

An admissible function $u$ is said to be a Jacobi function if the associated variation preserves the geodesic curvatures of each edge $C_{i j}$ and the angles in each vertex. The fact that the geodesic curvatures are preserved means that the restrictions $u_{i j}$ to $C_{i j}$ verify

$$
u_{i j}^{\prime \prime}+h_{i j}^{2} u_{i j}=0
$$

It is clear that the normal component of the Killing vector field generated by the rotations about the origin gives a Jacobi function.

From equation (1.7), we define the index form, that is, the induced bilinear form defined on the space of admissible functions, by

$$
\begin{aligned}
Q(u, v)=-\frac{1}{2}\left\{\sum_{\substack{i=1, \ldots, n \\
j \in I(i)}} \int_{C_{i j}}\left(u_{i j}^{\prime \prime}+h_{i j}^{2} u_{i j}\right) v_{i j}\right. \\
\left.\quad+\sum_{\substack{p \in \partial C_{i j} \\
p \in \operatorname{int}(D)}}\left(-q_{i j} u_{i j}+\frac{\partial u_{i j}}{\partial \nu_{i j}}\right)(p) v_{i j}(p)+\sum_{\substack{p \in \partial C_{i j} \\
p \in \partial D}}\left(u_{i j}+\frac{\partial u_{i j}}{\partial \nu_{i j}}\right)(p) v_{i j}(p)\right\},
\end{aligned}
$$

where $q_{i j}$ are the functions defined in Proposition 1.3.

We will say a stationary graph $C$ is stable if $Q(u, u) \geqslant 0$ for any admissible function $u$ whose associated variation preserves areas, and unstable if it is not stable. It is clear that a minimizing configuration must be stable. 
1.4. Existence and Regularity. From the results of F. Morgan M2, we obtain the following

Theorem 1.7 (Existence and Regularity M2 Th. 2.3]). Let $D \subset \mathbb{R}^{2}$ be a closed disk, and let $a_{1}, \ldots, a_{n}$ be $n$ given areas such that $\sum_{i=1}^{n} a_{i}=\operatorname{area}(D)$. Then there exists a graph separating $D$ into $n$ regions of areas $a_{1}, \ldots, a_{n}$. Moreover such a graph consists of constant geodesic curvature curves meeting in threes in the interior of $D$ at 120 degree angles, satisfying the balancing condition (1.3) for the geodesic curvatures, and meeting $\partial D$, one at a time, in an orthogonal way.

Proof. From the results in M2 one gets the existence of a solution and the regularity in the interior of the disk with just triple points as possible singularities. One also gets that there is a finite number of components (and hence of curves) in the minimizing configuration. For the boundary regularity, we only need to prove that at every point of $\partial D$, at most one curve of the minimizing configuration arrives, at 90 degrees.

If one or several curves meet $\partial D$ at $p$ and at least one of them is not orthogonal to $\partial D$, then the first variation formula implies that the graph is not stationary. Suppose now that several curves meet orthogonally $\partial D$ at $p$. We order them counter-clockwise and we consider the first one, $C$, which is the common boundary of components $\Omega_{i}$ and $\Omega_{j}$, with $\Omega_{i}$ a boundary one. Make a small deformation in the interior of $C$ which implies a loss of area $\delta$ for $\Omega_{i}$. In order to preserve the areas, it is possible to choose a point $q$ near $p$ in $C$, join $q$ to the second curve $C^{\prime}$, which is in the boundary of $\Omega_{j}$, and eliminate the part of $C$ between $p$ and $q$. It can be checked that this new configuration, for $\delta$ small enough, reduces perimeter. Then we get the desired regularity in the boundary of $D$.

1.5. Some properties of minimizing graphs. We now give and recall some results on minimizing graphs that will be used to prove our main theorem

Lemma 1.8. Given $n$ positive numbers $a_{1}, \ldots, a_{n}$ such that $\sum_{i=1}^{n} a_{i}=\pi$, we have

$$
I\left(a_{1}, \ldots, a_{n}\right) \leqslant n .
$$

Moreover, equality is never achieved for $n \geqslant 4$. If equality holds in the case $n=3$ then the standard graph consisting of three line segments dividing the disk into three regions of equal areas is minimizing.

Proof. We can divide the disk into regions of given areas $a_{1}, \ldots, a_{n}$ by using appropriate $n$ radii. This gives (1.9). For $n \geqslant 4$, this configuration has a prohibited singularity at the origin, so that it cannot be minimizing. If equality holds in (1.9) for $n=3$, the configuration must be stationary, so that the three radii meet in 120 degrees, and the configuration is the standard one for equal areas.

Lemma 1.9. A minimizing graph must be connected.

Proof. On a nonconnected graph, we can rotate one of the components until it touches another one creating an irregular meeting, so the graph cannot be minimizing.

Remark 1.10. Let $C \subset D$ be a minimizing graph, and $\Omega$ a connected component of $D-C$. Lemma 1.9 implies that $\partial \Omega \cap \partial D$ has to be connected.

Lemma 1.11 ([FABH, Lemma 2.4]). On a minimizing graph, there are no 2-components. 


\section{A BOUND ON THE NUMBER OF COMPONENTS OF THE LARGEST PRESSURE REGION}

Lemma 2.1. Let $C$ be a stable graph separating $D$ into $n$ regions. Then the region of largest pressure has at most $n-1$ nonhexagonal components.

Proof. Assume $R_{1}$ is the region of largest pressure and suppose it has at least $n$ nonhexagonal components, $\Omega_{1}, \ldots, \Omega_{n}$. For each $i$, consider the variation given by $u_{i}=1$ on $\partial \Omega_{i}$, extended by zero to the whole graph. If $\Omega_{i}$ is a boundary component then

$$
Q\left(u_{i}, u_{i}\right)=-\sum_{j \in I(1)}\left\{\int_{C_{1 j} \cap \partial \Omega_{i}} h_{1 j}^{2}+\sum_{\substack{p \in \partial C_{1 j} \cap \partial \Omega_{i} \\ p \in \operatorname{int}(D)}}-q_{1 j}(p)+\sum_{\substack{p \in \partial C_{1 j} \cap \partial \Omega_{i} \\ p \in \partial D}} 1\right\}<0,
$$

since, for $p$ in $C_{1 j} \cap C_{1 k} \cap \partial \Omega_{i}$, we have

$$
q_{1 j}(p)+q_{1 k}(p)=\frac{h_{k 1}+h_{k j}+h_{j 1}+h_{j k}}{\sqrt{3}}(p)=\frac{h_{k 1}+h_{j 1}}{\sqrt{3}}(p) \leqslant 0,
$$

as $R_{1}$ has the largest pressure.

If $\Omega_{i}$ is an interior component then $Q\left(u_{i}, u_{i}\right)$ can be computed as above except that the last summand does not appear. So we get

$$
Q\left(u_{i}, u_{i}\right) \leqslant 0
$$

and equality holds if and only if $\Omega_{i}$ is bounded by segments. It is easy to obtain, from GaussBonnet Theorem, that $\Omega_{i}$ has to be an hexagon. In the case of three regions, this only occurs if the three pressures are equal.

Hence, in our case we can find some nontrivial linear combination $u$ of $u_{i}$, such that the induced variation preserves areas up to first order and $Q(u, u)<0$.

Lemma 2.2. Let $C \subset D$ be a minimizing graph separating $D$ into three regions. Then $C$ is one of the graphs in Figure 4.

Proof. Suppose first that all the pressures are equal. If all the components touch the boundary of $D$ then $C$ is standard. If there is an interior component, then it is hexagonal. It is easy to see that the edges leaving the vertices of the hexagon meet $\partial D$ (otherwise we could find two different parallel rays meeting orthogonally $\partial D$ ). This implies that the graph is like in Figure 3. This graph has two regions with three nonhexagonal convex components, and so it is unstable by Lemma 2.1 .

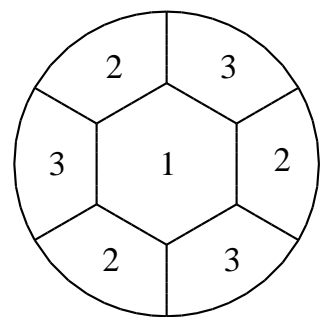

FiguRE 3. A graph with an hexagonal interior component 


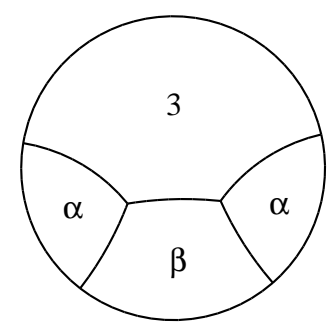

(1) $\alpha, \beta \in\{1,2\}$

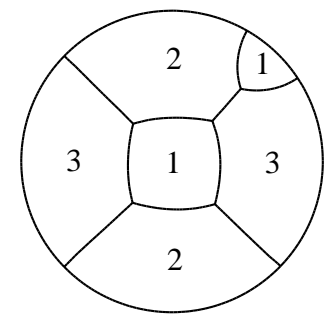

(4)

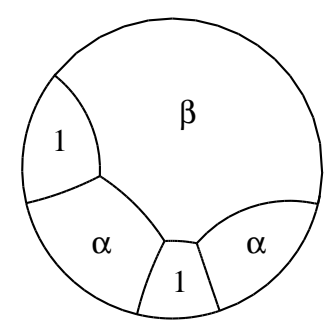

(7) $\alpha, \beta \in\{2,3\}$

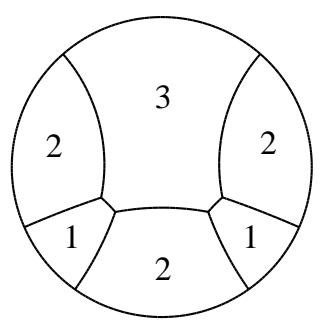

(2)

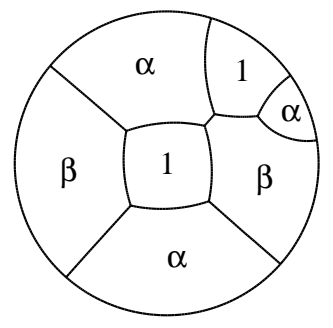

(5) $\alpha, \beta \in\{2,3\}$

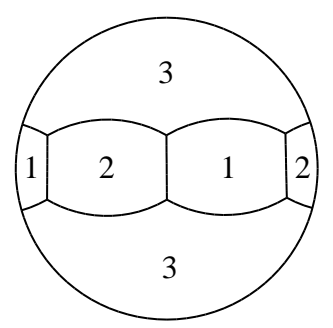

(8)

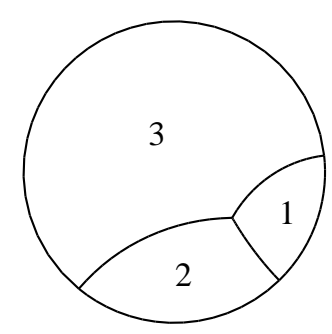

(10)

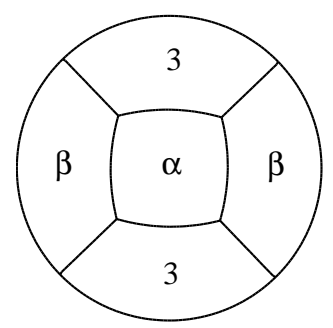

(3) $\alpha, \beta \in\{1,2\}$

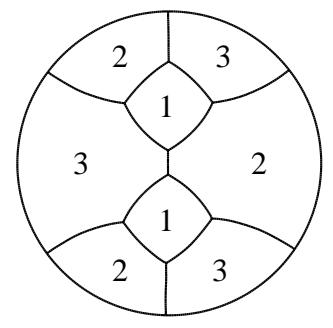

(6)

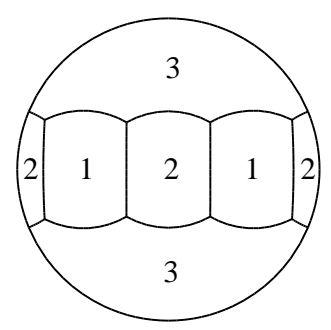

(9)

Figure 4. The ten possible configurations for minimizing graphs 
Assume now that $p_{1} \geqslant p_{2} \geqslant p_{3}$, with $p_{1}>p_{3}$. Then $R_{1}$ cannot have hexagonal components and so has at most two components, by Lemma 2.1. An interior component of $R_{1}$ has an even number of edges in its boundary and cannot be a 2-component by Lemma 1.11. So any interior component of $R_{1}$ is a quadrilateral. A boundary component of $R_{1}$ will have by Gauss-Bonnet Theorem three or four edges in its boundary.

Suppose $R_{1}$ is connected. If it touches $\partial D$ and has three edges, we have the standard configuration (10) and if it has four edges, we have configuration (1). If $R_{1}$ is interior, it will have only four edges, corresponding to configuration (3)

Suppose now $R_{1}$ has two connected components, $A$ and $B$. They can be interior or boundary components. We study each case.

If both are boundary components, as before, they can have three or four edges. If $A$ and $B$ have three edges, by the connectedness of $C$, the only possibility is configuration (1) If $A$ has four edges and $B$ has three edges, the only possibility is configuration [7) and if $A$ and $B$ have four edges, we will have configurations (2) and (3)

If $A$ is an exterior component and $B$ is an interior component, as above, $A$ will have three or four edges, and $B$ will have four. In the first case, we will get configuration (4) and in the second one, configurations (5) and (8)

If both components are interior, they will have four edges. A component of the region with the smallest pressure cannot be interior by Gauss-Bonnet Theorem. Then the only possibilities are configurations (6) and (9)

\section{UNSTABLE AND NON-MINIMIZING CONFIGURATIONS}

Lemma 3.1. Let $C_{12} \subset D$ be a circle or segment meeting $\partial D$ orthogonally between two regions $R_{1}^{\prime}, R_{2}^{\prime}$ with associated pressures $p_{1}$ and $p_{2}$ (the geodesic curvature of $C_{12} w . r$. $t$. the normal pointing into $R_{1}^{\prime}$ equals $p_{1}-p_{2}$ ).

Then, given $v \in C_{12}$, there exist unique curves $C_{23}, C_{31}$ with constant geodesic curvature yielding a standard graph. Moreover, the pressure $p_{3}$ of the third region is a monotone function of $v$ taking on all values between $-\infty$ and $+\infty$, and the regions $R_{3}^{v}$ are nested.

Proof. Fix $v \in C_{12}$. Let $q$ be a point in $C_{12} \cap \partial D$. Consider the Möbius transformation

$$
f(z)=\frac{i(z+q)}{q-z}
$$

which takes the disk $D$ to the upper half-plane and sends $q$ to infinity. Then $f\left(C_{12}\right)$ is a straight line $L$. Assume that there are $C_{23}, C_{31}$ curves with constant geodesic curvature meeting $C_{12}$ at $v$ so that $C_{12} \cup C_{23} \cup C_{31}$ is standard. Since $f$ is conformal, the sum of the geodesic curvatures of $f\left(C_{i j}\right)$ is zero. Moreover $f\left(C_{23}\right), f\left(C_{31}\right)$ intersect the real axis orthogonally. It is not difficult to see that $f\left(C_{23}\right), f\left(C_{31}\right)$ are circles centered at the real axis, with the same radius by the balancing condition (1.3). Thus $f\left(C_{23}\right), f\left(C_{31}\right)$ are unique and so are $C_{23}$ and $C_{31}$. It is clear that the regions determined by $f\left(C_{23}\right)$ and $f\left(C_{31}\right)$ are nested, which implies that $R_{3}^{v}$ are nested. 
Let $d$ be the distance from $f(v)$ to the real axis. By applying the inverse of $f$, it is possible to compute the geodesic curvatures $h_{31}$ and $h_{32}$ in terms of $d$, obtaining

$$
\begin{aligned}
& h_{31}=\frac{1}{4}\left(-\sqrt{3} d-2 x+\frac{\sqrt{3}\left(1+x^{2}\right)}{d}\right), \\
& h_{32}=\frac{1}{4}\left(-\sqrt{3} d+2 x+\frac{\sqrt{3}\left(1+x^{2}\right)}{d}\right) .
\end{aligned}
$$

Hence $p_{3}=p_{1}+h_{31}$, which decreases from $+\infty$ to $-\infty$ when $d$ moves from 0 to $+\infty$.

Proposition 3.2. Given three pressures, there is a standard graph separating the disk into three regions with the given pressures. Such a graph is unique up to a rigid motion of the disk.

Proof. Given two pressures $p_{1}, p_{2}$, there is a circle or segment $C_{12}$ separating $D$ into two regions with constant geodesic curvature $p_{1}-p_{2}$ meeting $\partial D$ orthogonally. $C_{12}$ is unique up to a rigid motion of the disk. Using Lemma 3.1 we can find a vertex $v \in C_{12}$ and unique curves $C_{23}, C_{31}$ so that $C_{12} \cup C_{23} \cup C_{31}$ is a standard graph separating the disk into three regions with the given pressures. Uniqueness follows from the construction.

Proposition 3.3. Let $C_{1}, C_{2}, C_{3}$ be circles or lines meeting at 120 degree angles at some interior point of $D$, satisfying the balancing condition (1.3). If $C_{1}$ and $C_{2}$ meet $\partial D$ orthogonally, then so it does $C_{3}$.

Proof. Let $\Omega$ be the region enclosed by $C_{1}, C_{2}$ and $\partial D$. Apply the disk onto the upper halfplane by means of a Möbius map $f$ sending $p \in \partial D-\partial \Omega$ to infinity. It is enough to show that $f\left(C_{3}\right)$ meets orthogonally the real axis. As in the proof of Lemma 3.1 $f\left(C_{1}\right)$ and $f\left(C_{2}\right)$ meet themselves at 120 degrees and the real axis at 90 degrees. As $f$ is a Möbius transformation, the balancing condition (1.3) is preserved. In case $f\left(C_{3}\right)$ is a line, it meets the real axis orthogonally. If $f\left(C_{3}\right)$ is a circle, then $f\left(C_{1}\right) \cup f\left(C_{2}\right) \cup f\left(C_{3}\right)$ is a standard planar double bubble, for which the centers of the circles are known to be aligned. The centers of $f\left(C_{1}\right)$ and $f\left(C_{2}\right)$ lie in the real axis, and hence also the center of $f\left(C_{3}\right)$. So we conclude that $f\left(C_{3}\right)$ meets the real axis orthogonally.

The proof of Lemma 3.1 establishes the existence of a deformation of one of the regions along one of the edges. More precisely we have

Proposition 3.4. Given a stationary graph $C$ with a boundary 3-component $\Omega$, there exists a variation of $C$ that

(i) strictly increases the pressure of $\Omega$ while keeping the other pressures unchanged, and

(ii) strictly decreases the area of $\Omega$, and

(iii) leaves invariant the edges of $C$ not lying in $\partial \Omega$.

Proposition 3.5. Let $C$ be a stationary graph in which a region has two boundary 3-components. Then $C$ is unstable.

Proof. Let $\Omega_{1}, \Omega_{2}$ be boundary 3-components of the same region. On each $\Omega_{i}$, consider the variation given by Proposition 3.4. The normal components $u_{i}$ of the associated variational vector fields have disjoint supports and satisfy $Q\left(u_{i}, u_{i}\right)<0$. By Propositions 3.2 and 3.3 , $\Omega_{1}$ 
and $\Omega_{2}$ are congruent so that $u=u_{1}-u_{2}$ satisfies the mean value conditions (1.1). Hence the graph is unstable.

Theorem 3.6. Given three areas $a_{1}, a_{2}, a_{3}$ such that $a_{1}+a_{2}+a_{3}=\operatorname{area}(D)$, there is a unique standard graph, up to rigid motions of the disk, separating $D$ into three regions of areas $a_{i}$.

Proof. Consider two standard graphs: $C=C_{12} \cup C_{23} \cup C_{31}$, and $C^{\prime}=C_{12}^{\prime} \cup C_{23}^{\prime} \cup C_{31}^{\prime}$ so that $a_{i}=\operatorname{area}\left(R_{i}\right)=\operatorname{area}\left(R_{i}^{\prime}\right), i=1,2,3$.

In case $h_{12}=h_{12}^{\prime}$ we can apply a rigid motion of the disk to $C$ until $C_{12}$ and $C_{12}^{\prime}$ coincide near $\partial D$. As the areas of the enclosed regions are equal, Lemma 3.1 implies that $C=C^{\prime}$.

Assume that $h_{12}>h_{12}^{\prime}$. By Lemma 3.1 we can continuously decrease the pressure $p_{1}$ (while keeping constant $p_{2}$ and $p_{3}$ ) until we get another standard graph $C^{\prime \prime}$ with $h_{12}^{\prime \prime}=h_{12}$. For this new graph $C^{\prime \prime}$ we get $\operatorname{area}\left(R_{1}^{\prime \prime}\right)>a_{1}$, area $\left(R_{i}^{\prime \prime}\right)<a_{i}$, for $i=2,3$. Now we can apply an isometry of the disk to $C^{\prime \prime}$ so that $C_{12}^{\prime \prime}$ and $C_{12}^{\prime}$ coincide near $\partial D$. As area $\left(R_{1}^{\prime \prime}\right)>a_{1}$, Lemma 3.1 implies that area $\left(R_{2}^{\prime \prime}\right)>a_{2}$, which gives us a contradiction.

Given two edges in a graph $C$, we will say that they are cocircular if they have the same center. A cocircular 4-component will be a 4-component with two cocircular opposite edges.

Remark 3.7. It is easy to check that in a sequence of interior 4-components, if any of them is cocircular, then all the 4-components are.

Lemma 3.8 (㐭, Lemma 5.38]). Suppose we have a stationary graph with a sequence of at least three cocircular 4-components, so that the first and the last are boundary components and the remaining are interior ones (cocircularity refers to the edges of the boundary components meeting $\partial D)$. Assume further that the components out of the chain belong to the same region. Then there is a continuous movement preserving perimeter and areas which creates an illegal meeting, so that the graph is not minimizing.

Proof. Order the 4-components so that $\Omega_{1}$ and $\Omega_{n}$ are the boundary ones, and $\Omega_{i}$ meets $\Omega_{i+1}$. Let $c_{i}$ be the center of the cocircular arcs of $\Omega_{i}$. We can move these points, without changing neither $d\left(c_{i}, c_{i+1}\right)$ nor $d\left(c_{1}, 0\right)$ and $d\left(c_{n}, 0\right)$ in such a way that $c_{1}$ and $c_{n}$ get closer and closer. With this movement of the points, we obtain a deformation of the graph which preserves perimeter and the areas of the regions and will create an irregular meeting. Hence the graph cannot be minimizing.

Lemma 3.9 (W Lemma 5.3]). Let e, $f$ and $g$ be three consecutive edges of a component, and let $v_{1}$ and $v_{2}$ be the corresponding vertices. Suppose e and $g$ have the same geodesic curvature, and the angles in each vertex are the same. Let $R$ be the line of points equidistant from $v_{1}$ and $v_{2}$.

Then e and $g$ are interchanged by the symmetry about $R$. Moreover, if e and $g$ are cocircular, the common center lies in $R$, and if $e$ and $g$ are not cocircular, $R$ coincides with the line of points equidistant from the centers of $e$ and $g$.

Given a graph $C$ and a Jacobi function $u$ defined on it, we will say that a point $x$ in $C$ is a nodal point if $u(x)=0$. A nodal region will be a connected component of the complementary in $C$ of the set of nodal points. 
Proposition 3.10 ([HMRR, Proposition 5.2]). Let $C$ be a stationary graph separating the disk into three regions. Assume that there exists a Jacobi function with at least four nodal regions such that the nodal points are not vertices of the graph. Then $C$ is unstable.

Proof. Let $u$ be the Jacobi function, and $N_{1}, \ldots, N_{4}$ nodal regions. Assume that the graph $C$ is stable. For $i=1, \ldots, 4$ define $u_{i}$ as the restrictions of $u$ to $N_{i}$ extended by zero to the whole graph. It is possible to find a nontrivial linear combination $v$ of $u_{1}, u_{2}, u_{3}$ so that the mean value conditions (1.1) are satisfied for $R_{1}, R_{2}$ and $R_{3}$. Moreover, $v$ is an admissible function and, by stability, is a Jacobi function. As $v$ vanishes on a subset of $C$ containing $N_{4}$ and has nontrivial support contained in $N_{1} \cup N_{2} \cup N_{3}$, there is an edge $\ell$ so that $v$ vanishes on an open interval of $\ell$ but it is not identically zero on $\ell$. As $v$ is a Jacobi function (a solution of a second order o.d.e.) this gives us a contradiction.

Now we discard configuration (8) by a geometrical argument. The reader may compare this result with CHLL, Lemma 5.2.10]

Proposition 3.11. Configuration (8) is not minimizing.
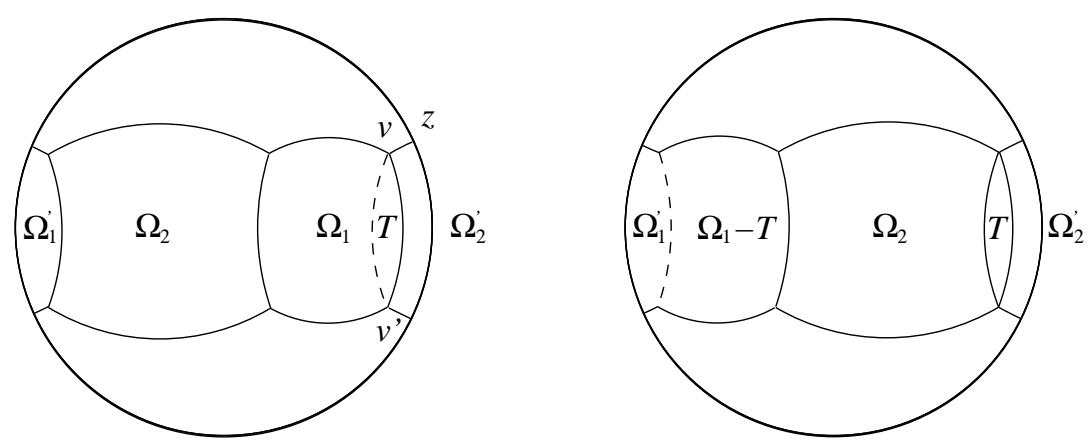

Figure 5. Deformation of a chain of symmetric 4-components obtaining a graph with irregular vertices

Proof. Suppose this configuration is minimizing. We will denote by $\Omega_{1} \subset R_{1}, \Omega_{2} \subset R_{2}$ the interior components, and by $\Omega_{1}^{\prime} \subset R_{1}, \Omega_{2}^{\prime} \subset R_{2}$ the boundary components.

Consider any component of $R_{1}$ or $R_{2}$ and suppose the edges separating such a component and $R_{3}$ are cocircular. By Remark 3.7 we will have a sequence of four cocircular 4-components and using Lemma 3.8 the configuration is not minimizing.

Hence the considered edges cannot be cocircular, and applying Lemma 3.9, it is easy to check that there exists a horizontal symmetry of the chain of 4-components which is in fact a symmetry of the whole configuration.

In each interior component, using Lemma 3.9 and taking into account that any pair of opposite edges cannot be cocircular, it can be seen that there exists a vertical symmetry, orthogonal to the horizontal one.

If $p_{1}=p_{2}$, we have that $\Omega_{1}$ and $\Omega_{2}$ are congruent, so that they have the same area. Hence, we can interchange them, and after eliminating unnecessary edges, we obtain a new configuration enclosing the same areas with strictly less perimeter, which gives a contradiction. 
If $p_{2}=p_{3}$, applying Gauss-Bonnet Theorem to $\Omega_{2}$ we obtain a contradiction.

So we can suppose now $p_{1}>p_{2}>p_{3}$. Let us distinguish two cases:

Let $z$ be the upper point in $\partial \Omega_{2}^{\prime} \cap \partial D$. Let $\theta$ be the angle between the line $0 z$ and a horizontal line. Assume that $\theta$ is greater than or equal to $\pi / 4$. Then, the second coordinate of $z$ will be greater than or equal to $\cos \pi / 4=\sqrt{2} / 2$. We can see $\partial \Omega_{2}^{\prime}$ as a vertical graph, so the length of $\partial \Omega_{2}^{\prime}$ will be greater than or equal to $\sqrt{2}$. By similar arguments, taking into account that the point of $\partial \Omega_{2}$ with maximum second coordinate will be higher than $z$, the length of $\partial \Omega_{2}$ will be greater than or equal to $2 \sqrt{2}$. Both quantities add up to more than 3 , so by Lemma 1.8, this configuration cannot be minimizing.

Assume now that $\theta$ is less than $\pi / 4$. Let $l$ be the edge separating $\Omega_{1}$ and $\Omega_{2}^{\prime}$, and $v, v^{\prime}$ its vertices. Consider a new edge $\widetilde{l}$, the symmetric of $l$ about the segment $\overline{v v^{\prime}}$, and let $T$ be the region enclosed between $l$ and $\widetilde{l}$. Move $\Omega_{2}$ in the horizontal direction until one of its edges coincides with $\widetilde{l}$. Reflect $\Omega_{1}-T$ about a vertical axis so that the reflection of $\widetilde{l}$ coincides with the edge $\Omega_{2} \cap \Omega_{1}^{\prime}$ (recall that each interior 4-component has a vertical symmetry, so the side edges have the same length and curvature). After eliminating an unnecessary edge, this new configuration will preserve length and areas, but it will be irregular. Hence, configuration (8) cannot be minimizing. It only remains to check that $\Omega_{1}$ and $\Omega_{2}$ will remain inside $D$ under this geometrical transformation.

In order to prove this it is enough to show that the portion of the original graph over the horizontal line $L$ passing through $z$ stays inside $D$. The portion $\ell_{2}$ of the upper edge of $\Omega_{2}$ over $L$ makes an angle $\theta$ with $L$, and can be translated horizontally to touch $z$ at its boundary. Since $\theta<\pi / 4, \ell_{2}$ must lie inside $D$. This implies that the transformation of $\Omega_{2}$ stays inside $D$. The upper edge $\ell_{1}$ of $\Omega_{1}$ has larger geodesic curvature than the upper edge of $\Omega_{2}$ and makes a smaller angle with the horizontal line passing through $v$. This is enough to conclude that $\ell_{1}$ must lie inside $D$.

\section{Proof of the theOREM}

In this section we prove the main theorem.

Theorem 4.1. Let $C \subset D$ be a minimizing graph for three given areas. Then $C$ is a unique standard graph.

Proof. By Lemma 2.2 the graph $C$ must be one of the listed in Figure 4 .

Configurations (1) and (2) are unstable by Proposition 3.5.

Configuration (3) is also unstable: it is easy to check that the edges of $C_{12}$ are not cocircular, so that by Remark 3.7 and Lemma 3.9. we have a vertical symmetry in this configuration. By Lemma 3.8 the edges of $C_{13}$ cannot be cocircular if $C$ is minimizing. Applying again Lemma 3.9 we get an horizontal symmetry. Both axes of symmetry will meet orthogonally at the origin, so we can consider the Killing field generated by rotations about 0 , which yields a Jacobi function $u$ vanishing on four points, one on the interior of each edge of the central 4-component. So $u$ has at least four nodal regions and we conclude by Proposition 3.10 that $C$ is unstable. 
We now eliminate configurations (4) and (5). These configurations present an interior 4-component of $R_{1}$, with three incident edges meeting the exterior of the disk. If we extend the fourth edge, it will meet $\partial D$ orthogonally by Proposition 3.3 and we will obtain a configuration of type (3). Hence, the interior 4-component has two orthogonal symmetries meeting at the origin, and we conclude as before the existence of four nodal regions.

Consider now configuration (6) Fix an interior 4-component $\Omega$ of $R_{1}$. If we extend the edge leaving the boundary of $\Omega$ that does not reach $\partial D$, it will meet $\partial D$ orthogonally due to the existence of a symmetry of $\Omega$ which is in fact a symmetry of the disk. In this way we obtain a configuration of type (3). As above, $\Omega$ will have two orthogonal symmetries meeting at 0 and so we can get four nodal regions yielding instability.

Consider now configuration (7)] Applying Lemma 3.9 the two 4-components will be symmetric about two lines $r_{1}, r_{2}$ passing through the center of the disk (the corresponding edges are not cocircular). Let $q_{1}, q_{2}$ be the intersection points of each line with the interior edges of these components, that will be zeros of the Jacobi field $u$ induced by the oneparameter group of rotations about the origin. The reflection of $q_{1}$ with respect to $r_{2}$ lies in the boundary of the 3-component of $R_{1}$ and it is not a vertex of the configuration. This point is clearly also a zero of $u$. Then $u$ has four nodal regions and the configuration is unstable.

By Proposition 3.11, configuration (8) is not minimizing.

Configuration (9) is unstable: if the top and bottom edges of each component are cocircular then the configuration is not minimizing by Lemma 3.8. Otherwise we can find an horizontal symmetry of the graph, which is also a symmetry of the disk by Lemma 3.9. Each interior 4component has a vertical symmetry so that the interior components of $R_{1}$ are identical. Using the function equal to +1 on one of these components, equal to -1 on the other component, and zero otherwise, we have obtained a function satisfying the mean value conditions (1.1) which is negative for the index form. So this configuration is unstable. We could also use the method of Proposition 3.11 to see that this configuration is nonminimizing.

So the only remaining possibility is configuration (10) the standard one. Uniqueness for given areas comes from Theorem 3.6

\section{FinAL REMARKS}

In this paper we have obtained that the problem of dividing the disk into three areas has a unique solution in which all regions are connected, as in the problem of partitioning the disk into two areas. It is natural to conjecture that

Conjecture 5.1. A minimizing graph separates the disk into connected regions.

If we consider the problem for $n$ regions, with $n \geqslant 4$, by Lemma 2.1 the region of largest pressure will have at most $n-1$ nonhexagonal connected components and we can obtain by combinatorial arguments a list of all possible minimizing configurations. Of course the number of candidates increases very rapidly when the number of regions increases. We believe that the following conjectures are true

Conjecture 5.2. The least perimeter way of dividing the unit disk into four regions of prescribed areas is given by configuration (1) of Figure 6 . 
Conjecture 5.3. The least perimeter way of dividing the unit disk into five regions of prescribed areas is given by configuration (2) of Figure 6 .

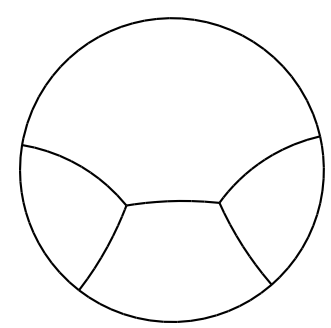

(1)

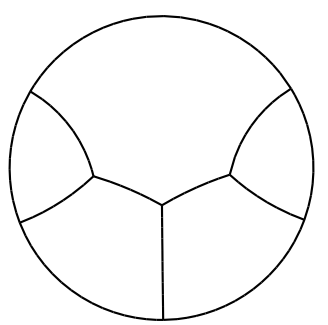

(2)

Figure 6. The conjectural configurations for $n=4$ and $n=5$

For each case, we believe that there is another possibly stable configuration: for $n=4$, the configuration with three boundary regions surrounding an interior one of three edges, and for $n=5$, the one consisting in four boundary regions surrounding an interior region of four edges. But estimates we have done using Surface Evolver (Ken Brakke, 1992) for equal areas show that they are nonminimizing. Furthermore, for $n=4$, if any of the areas tends to zero, we should obtain in the limit the standard configuration for three areas, which also discards the configuration described above at least for some areas. In the case $n=5$ we should have the same behaviour.

For $n=6$ we give the following conjecture

Conjecture 5.4. The least perimeter way of dividing the unit disk into six regions of prescribed areas is given by configuration of Figure 7.

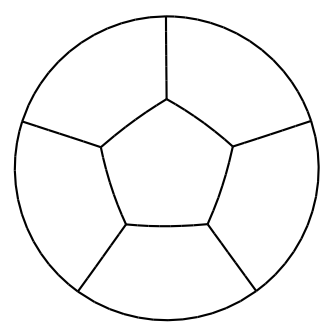

FiguRE 7. The conjectural configuration for $n=6$

As before, we belive that the configurations of Figure 8 below are stable, but estimates done with the Surface Evolver considering equal areas show that they are nonminimizing. 


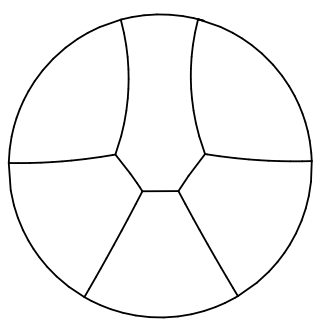

(1)

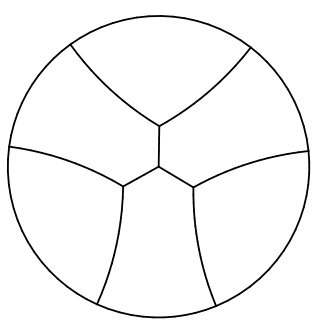

$(2)$

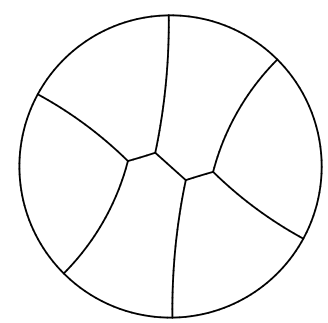

(3)

FiguRE 8. Some other configurations for $n=6$

\section{REFERENCES}

[Alm] F. J. Almgren, Jr., Existence and regularity almost everywhere of solutions to elliptic variational problems with constraints, Bull. Amer. Math. Soc. 81 (1975), 151-154. MR 50 \#14438

[B1] M. N. Bleicher, Isoperimetric divisions into several cells with natural boundary, Intuitive geometry (Siófok, 1985), Colloq. Math. Soc. János Bolyai, vol. 48, North-Holland, Amsterdam, 1987, pp. 6384. MR 88j:52023

[B2] Isoperimetric networks in the Euclidean plane, Studia Sci. Math. Hungar. 31 (1996), no. 4, 455-478. MR 96k:52014

[B3] Isoperimetric division into a finite number of cells in the plane, Studia Sci. Math. Hungar. 22 (1987), no. 1-4, 123-137. MR 89a:52036

[BL] Tracy Borawski and Robert Lopez, The double bubble problem on the cone, preprint.

[CCWB] Miguel Carrión Álvarez, Joseph Corneli, Genevieve Walsh, and Shabnam Beheshti, Double Bubbles in the Three-Torus, Experiment. Math., to appear.

[CHLL] Joseph Corneli, Paul Holt, George Lee, Nicholas Leger, Eric Schoenfeld, and Benjamin Steinhurst, The double bubble problem on the flat two-torus, preprint.

[CF] Andrew Cotton and David Freeman, The double bubble problem in spherical space and hyperbolic space, Int. J. Math. Math. Sci. 32 (2002), no. 11, 641-699. MR 1949693

[CHH] Christopher Cox, Lisa Harrison, Michael Hutchings, and et al., The shortest enclosure of three connected areas in $\mathbb{R}^{2}$, Real Anal. Exchange 20 (1994/95), no. 1, 313-335. MR 95k:53009

[CGFM] S.J. Cox, F. Graner, M. Fátima Vaz, C. Monnereua-Pittet, and N. Pittet, Minimal perimeter for $N$ identical bubbles in two dimensions: calculations and simulations, Philosophical Magazine 83 (2003), 1393-1406.

[D] Richard Paul Devereaux Vaughn, Planar Soap Bubbles, Ph D thesis, University of California, Davis, 1998.

[FABH] Joel Foisy, Manuel Alfaro, Jeffrey Brock, Nickelous Hodges, and Jason Zimba, The standard double soap bubble in $\mathbf{R}^{2}$ uniquely minimizes perimeter, Pacific J. Math. 159 (1993), no. 1, 47-59. MR 94b:53019

[GJJF] F. Graner, Y. Jiang, E. Janiaud, and C. Flament, Equilibrium energies of 2D fluid foams, Phys. Rev. E. 63 (2001), 11402.

[HS] Joel Hass and Roger Schlafly, Double bubbles minimize, Ann. of Math. (2) 151 (2000), no. 2, 459515. MR 2002d:53018

[HLPS] G. Christopher Hruska, Dmitriy Leykekhman, Daniel Pinzon, Brian J. Shay, and Joel Foisy, The shortest enclosure of two connected regions in a corner, Rocky Mountain J. Math. 31 (2001), no. 2, 437-482. MR 2002h:53008

[HMRR] Michael Hutchings, Frank Morgan, Manuel Ritoré, and Antonio Ros, Proof of the double bubble conjecture, Ann. of Math. (2) 155 (2002), no. 2, 459-489. MR 2003c:53013

[M] Joseph D. Masters, The perimeter-minimizing enclosure of two areas in $S^{2}$, Real Anal. Exchange 22 (1996/97), no. 2, 645-654. MR 99a:52010 
[M1] Frank Morgan, (M, $\epsilon, \delta)$-minimal curve regularity, Proc. Amer. Math. Soc. 120 (1994), no. 3, 677686. MR 94e: 49018

[M2] _ Soap bubbles in $\mathbb{R}^{2}$ and in surfaces, Pacific J. Math. 165 (1994), no. 2, 347-361. MR 96a:58064

[MW] Frank Morgan and Wacharin Wichiramala, The standard double bubble is the unique stable double bubble in $\mathbb{R}^{2}$, Proc. Amer. Math. Soc. 130 (2002), no. 9, 2745-2751 (electronic). MR 2003c:53016

[RHLS] Ben W. Reichardt, Cory Heilmann, Yuan Y. Lai, and Anita Spielman, Proof of the Double Bubble Conjecture in $\mathbb{R}^{4}$ and certain higher dimensional cases, Pac. J. Math. 208 (2003), 347-366.

[Th] D'Arcy Wentworth Thompson, On Growth and Form: The Complete Revised Edition, Dover Publications, 2002.

[W] Wacharin Wichiramala, The Planar Triple Bubble Problem, Ph. D. Thesis, University of Illinois, Urbana-Champaign, 2002.

Departamento de Geometría y Topología, Facultad de Ciencias, Universidad de Granada, E-18071 GranAdA (ESPAÑA)

E-mail address: antonioc@ugr.es

Departamento de Geometría y Topología, Facultad de Ciencias, Universidad de Granada, E-18071 Granada (EsPaña)

E-mail address: ritore@ugr.es 